\title{
Three-Nucleon Forces explored by Lattice QCD Simulations
}

\section{Takumi Doi*}

Center for Nuclear Study, The University of Tokyo, Tokyo 113-0033, Japan

E-mail: doidribf.riken.ip

\section{for HAL QCD Collaboration}

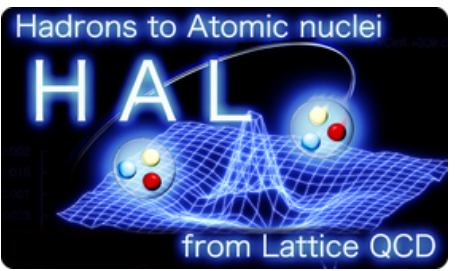

We explore three-nucleon forces (3NF) from lattice QCD simulations. Utilizing the NambuBethe-Salpeter (NBS) wave function, two-nucleon forces (2NF) and $3 \mathrm{NF}$ are determined on the same footing. Quantum numbers of the three-nucleon $(3 \mathrm{~N})$ system are chosen to be $\left(I, J^{P}\right)=$ $\left(1 / 2,1 / 2^{+}\right)$(the triton channel). The enormous computational cost is reduced by employing the simplest geometrical configuration, where $3 \mathrm{~N}$ are aligned linearly with an equal spacing. We perform lattice QCD simulations using $N_{f}=2$ dynamical clover fermion configurations generated by CP-PACS Collaboration, at the lattice spacing of $a=0.156 \mathrm{fm}$ on a $16^{3} \times 32$ lattice with a large quark mass corresponding to $m_{\pi}=1.13 \mathrm{GeV}$. Repulsive $3 \mathrm{NF}$ is found at short distance.

The XXIX International Symposium on Lattice Field Theory - Lattice 2011

July 10-16, 2011

Squaw Valley, Lake Tahoe, California

\footnotetext{
* Speaker.
} 


\section{Introduction}

Determination of the properties of three-nucleon forces (3NF) is one of the most important issue in nuclear physics and astrophysics these days. There are various phenomena where $3 \mathrm{NF}$ are considered to play an important role, e.g., the binding energies of light nuclei [U], the properties of neutron-rich nuclei and the supernova nucleosynthesis [ [ $]$, the nuclear equation of state (EoS) at high density relevant to the physics of neutron stars [3], 团] and the cross sections in nucleus-nucleus elastic scatterings [ []]. Deuteron-proton elastic scattering experiments at intermediate energies have also shown a clear indication of $3 \mathrm{NF}$ [焑].

Despite of its phenomenological importance, microscopic understanding of 3NF is still limited. Pioneered by Fujita and Miyazawa $[\square]$, the long range part of 3NF has been commonly modeled by the two-pion exchange $(2 \pi \mathrm{E})$, particularly with the $\Delta$-resonance excitation. An additional repulsive component of $3 \mathrm{NF}$ at short distance is often introduced in a purely phenomenological way [ []$]$. An approach based on the chiral effective field theory is also developing [ $[\mathrm{Q}]$.

However, since $3 \mathrm{NF}$ is originated by the fact that a nucleon is not a fundamental particle, it is most desirable to determine $3 \mathrm{NF}$ directly from the fundamental degrees of freedom (DoF), i.e., quarks and gluons, on the basis of quantum chromodynamics (QCD). In this proceeding, we carry out first-principle calculations of 3NF using lattice QCD simulations. Note that while there are lattice QCD works for three- and four- baryon systems [ए], प]], they focus on the energies of the multi-baryon systems, and extracting $3 \mathrm{NF}$ is currently beyond their scope.

As for the calculation of two-nucleon forces (2NF) from lattice $\mathrm{QCD}$, an approach based on the NBS wave function has been proposed [12, [3]], so that the potential is faithful to the phase shift by construction. Resultant (parity-even) 2NF are found to have desirable features such as attractive wells at long and medium distances and central repulsive cores at short distance. The method has been successfully extended to the hyperon-nucleon (YN) and hyperon-hyperon (YY)

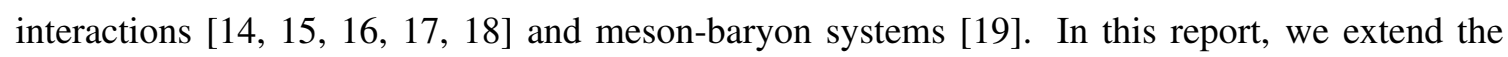
method to the three-nucleon $(3 \mathrm{~N})$ system, and perform the lattice QCD simulations for $3 \mathrm{NF}$ in the triton channel, $\left(I, J^{P}\right)=\left(1 / 2,1 / 2^{+}\right)[[20$, 2]1, [22, [2] ]. For details of this study, refer to Ref. [[2]].

\section{Formalism}

The detailed formulation for the determination of $2 \mathrm{NF}$ is given in Ref. [[प3], and we here focus on the extension to the $3 \mathrm{~N}$ system. We consider the NBS wave function of the $3 \mathrm{~N}, \psi_{3 N}(\vec{r}, \vec{\rho})$, extracted from the six-point correlator as

$$
\begin{aligned}
G_{3 N}\left(\vec{r}, \vec{\rho}, t-t_{0}\right) & \equiv \frac{1}{L^{3}} \sum_{\vec{R}}\left\langle 0\left|\left(N\left(\vec{x}_{1}\right) N\left(\vec{x}_{2}\right) N\left(\vec{x}_{3}\right)\right)(t) \overline{\left(N^{\prime} N^{\prime} N^{\prime}\right)}\left(t_{0}\right)\right| 0\right\rangle \\
& \underset{t \gg t_{0}}{\longrightarrow} A_{3 N} \psi_{3 N}(\vec{r}, \vec{\rho}) e^{-E_{3 N}\left(t-t_{0}\right)} \\
\psi_{3 N}(\vec{r}, \vec{\rho}) & \equiv\left\langle 0\left|N\left(\vec{x}_{1}\right) N\left(\vec{x}_{2}\right) N\left(\vec{x}_{3}\right)\right| E_{3 N}\right\rangle, \quad A_{3 N} \equiv\left\langle E_{3 N}\left|\overline{\left(N^{\prime} N^{\prime} N^{\prime}\right)}\right| 0\right\rangle
\end{aligned}
$$

where $E_{3 N}$ and $\left|E_{3 N}\right\rangle$ denote the energy and the state vector of the $3 \mathrm{~N}$ ground state, respectively, $N$ $\left(N^{\prime}\right)$ the nucleon operator in the sink (source), and $\vec{R} \equiv\left(\vec{x}_{1}+\vec{x}_{2}+\vec{x}_{3}\right) / 3, \vec{r} \equiv \vec{x}_{1}-\vec{x}_{2}, \vec{\rho} \equiv \vec{x}_{3}-\left(\vec{x}_{1}+\right.$ $\left.\vec{x}_{2}\right) / 2$ the Jacobi coordinates. 
With the derivative expansion of the potentials [24], the NBS wave function can be converted to the potentials through the following Schrödinger equation,

$$
\left[-\frac{1}{2 \mu_{r}} \nabla_{r}^{2}-\frac{1}{2 \mu_{\rho}} \nabla_{\rho}^{2}+\sum_{i<j} V_{2 N}\left(\vec{r}_{i j}\right)+V_{3 N F}(\vec{r}, \vec{\rho})\right] \psi_{3 N}(\vec{r}, \vec{\rho})=E_{3 N} \psi_{3 N}(\vec{r}, \vec{\rho}),
$$

where $V_{2 N}\left(\vec{r}_{i j}\right)$ with $\vec{r}_{i j} \equiv \vec{x}_{i}-\vec{x}_{j}$ denotes the $2 \mathrm{NF}$ between $(i, j)$-pair, $V_{3 N F}(\vec{r}, \vec{\rho})$ the $3 \mathrm{NF}, \mu_{r}=$ $m_{N} / 2, \mu_{\rho}=2 m_{N} / 3$ the reduced masses. If we calculate $\psi_{3 N}(\vec{r}, \vec{\rho})$ for all $\vec{r}$ and $\vec{\rho}$, and if all $V_{2 N}\left(\vec{r}_{i j}\right)$ are obtained by (separate) lattice calculations for genuine $2 \mathrm{~N}$ systems, we can extract $V_{3 N F}(\vec{r}, \vec{\rho})$ through Eq. (2.4).

In practice, however, the computational cost is enormous, because of enlarged color/spinor DoF by the $3 \mathrm{~N}$ (i.e., 9 valence quarks) and factorial number of the Wick contractions. In order to reduce the cost, we first take advantage of symmetries (such as isospin symmetry) to reduce the number of the Wick contractions. Second, we utilize that there is a freedom for the choice of a nucleon interpolating operator. In particular, a potential is independent of the choice of a nucleon operator at the source, $N^{\prime}$, and we employ the non-relativistic limit operator as $N^{\prime}=N_{n r} \equiv$ $\varepsilon_{a b c}\left(q_{a}^{T} C \gamma_{5} P_{n r} q_{b}\right) P_{n r} q_{c}$ with $P_{n r}=\left(1+\gamma_{4}\right) / 2$. Compared to the standard nucleon operator, $N_{s t d} \equiv$ $\varepsilon_{a b c}\left(q_{a}^{T} C \gamma_{5} q_{b}\right) q_{c}$, the spinor DoF of each (source) nucleon is reduced by half, and the computational cost of the $3 \mathrm{~N}$ system is reduced by a factor of $2^{3}=8$. On the other hand, a potential is dependent on the choice of a nucleon operator at the sink, $N$, since a NBS wave function is defined through a sink operator. Note, however, that physical observables calculated from these different potentials, such as phase shifts and binding energies, are unique by construction [[3]. In this sense, choosing $N$ corresponds to choosing the "scheme" to define the potential [123, [25]. In Ref. [24], it is found that the non-locality of 2NF from the choice of $N=N_{s t d}$ is small, and $N=N_{s t d}$ can be considered to be a "good scheme". We therefore employ the same sink operator $N=N_{s t d}$ in the $3 \mathrm{~N}$ study as well, so that $2 \mathrm{NF}$ and $3 \mathrm{NF}$ are determined on the same footing. Finally, we restrict the geometry of the $3 \mathrm{~N}$. More specifically, we consider the "linear setup"with $\vec{\rho}=\overrightarrow{0}$, with which $3 \mathrm{~N}$ are aligned linearly with equal spacings of $r_{2} \equiv|\vec{r}| / 2$. In this setup, the third nucleon is attached to $(1,2)$-nucleon pair with only S-wave. Considering the total $3 \mathrm{~N}$ quantum numbers of $\left(I, J^{P}\right)=\left(1 / 2,1 / 2^{+}\right)$, the triton channel, the wave function can be completely spanned by only three bases, which can be labeled by the quantum numbers of $(1,2)$-pair as ${ }^{1} S_{0},{ }^{3} S_{1},{ }^{3} D_{1}$. Therefore, the Schrödinger equation leads to the $3 \times 3$ coupled channel equations with the bases of $\psi_{1} S_{0}, \psi_{{ }^{3}} S_{1}, \psi_{3^{3} D_{1}}$. The reduction of the dimension of bases is expected to improve the $\mathrm{S} / \mathrm{N}$ as well. It is worth mentioning that considering the linear setup is not an approximation: Among various geometric components of the wave function of the ground state in the triton channel, we calculate the (exact) linear setup component as a convenient choice to study $3 \mathrm{NF}$. While we can access only a part of $3 \mathrm{NF}$ from it, we plan to extend the calculation to more general geometries step by step, toward the complete determination of the full $3 \mathrm{NF}$.

We consider the identification of genuine 3NF. It is a nontrivial work: Although both of parityeven and parity-odd 2NF are required to subtract 2NF part in Eq. (2.4), parity-odd 2NF have not been obtained yet in lattice QCD. (See, however, our recent progress on this issue [26].) In order to resolve this issue, we consider the following channel,

$$
\psi_{S} \equiv \frac{1}{\sqrt{6}}\left[-p_{\uparrow} n_{\uparrow} n_{\downarrow}+p_{\uparrow} n_{\downarrow} n_{\uparrow}-n_{\uparrow} n_{\downarrow} p_{\uparrow}+n_{\downarrow} n_{\uparrow} p_{\uparrow}+n_{\uparrow} p_{\uparrow} n_{\downarrow}-n_{\downarrow} p_{\uparrow} n_{\uparrow}\right]
$$


which is anti-symmetric in spin/isospin spaces for any $2 \mathrm{~N}$-pair. Combined with the Pauli-principle, it is automatically guaranteed that any $2 \mathrm{~N}$-pair couples with even parity only. Therefore, we can extract $3 \mathrm{NF}$ unambiguously using only parity-even 2NF. Note that no assumption on the choice of 3D-configuration of $\vec{r}, \vec{\rho}$ is imposed in this argument, and we thus can take advantage of this feature for future $3 \mathrm{NF}$ calculations with various setup of 3D-geometries.

\section{Lattice QCD setup and Numerical results}

We employ $N_{f}=2$ dynamical configurations with mean field improved clover fermion and RG-improved gauge action generated by CP-PACS Collaboration [D2]. We use 598 configurations at $\beta=1.95$ and the lattice spacing of $a^{-1}=1.269(14) \mathrm{GeV}$, and the lattice size of $V=L^{3} \times T=$ $16^{3} \times 32$ corresponds to $(2.5 \mathrm{fm})^{3}$ box in physical spacial size. For $u, d$ quark masses, we take the hopping parameter at the unitary point as $\kappa_{u d}=0.13750$, which corresponds to $m_{\pi}=1.13 \mathrm{GeV}$, $m_{N}=2.15 \mathrm{GeV}$ and $m_{\Delta}=2.31 \mathrm{GeV}$. We use the wall quark source with Coulomb gauge fixing. In order to enhance the statistics, we perform the measurement on 32 wall sources using different time slices, and the forward and backward propagations are averaged. The results from both of total angular momentum $J_{z}= \pm 1 / 2$ are averaged as well. Due to the enormous computational cost, we can perform the simulations only at a few sink time slices. Looking for the range of sink time where the ground state saturation is achieved, we carry out preparatory simulations for effective $2 \mathrm{NF}$ in the $3 \mathrm{~N}$ system in the triton channel at $2 \leq\left(t-t_{0}\right) / a \leq 11$, and find that the results are consistent with each other as long as $\left(t-t_{0}\right) / a \geq 7$ [22]. Being on the safer side, we perform linear setup calculations of $3 \mathrm{NF}$ at $\left(t-t_{0}\right) / a=8$ and 9 . We perform the simulation at eleven physical points of the distance $r_{2}$.

In Fig. 皿, we plot the radial part of each wave function of $\psi_{S}=\left(-\psi_{1_{1}}+\psi_{3 S_{1}}\right) / \sqrt{2}, \psi_{M} \equiv$ $\left(\psi_{1} S_{0}+\psi_{3_{1}}\right) / \sqrt{2}$ and $\psi_{3^{3} D_{1}}$ obtained at $\left(t-t_{0}\right) / a=8$. Here, we normalize the wave functions by the central value of $\psi_{S}\left(r_{2}=0\right)$. What is noteworthy is that the wave functions are obtained with good precision, which is quite nontrivial for the $3 \mathrm{~N}$ system. We observe that $\psi_{S}$ overwhelms other wave functions. This indicates that higher partial wave components are strongly suppressed, and thus the effect of the next leading order in the derivative expansion, spin-orbit forces, is suppressed in this lattice setup.

We determine $3 \mathrm{NF}$ by subtracting $2 \mathrm{NF}$ from total potentials in the $3 \mathrm{~N}$ system. Since we have only one channel (Eq. (L.5 ) ) which is free from parity-odd 2NF, we can determine one type of 3NF. In this report, $3 \mathrm{NF}$ are effectively represented in a scalar-isoscalar functional form, which is often employed for the short-range 3NF in phenomenology [ [ $]$ ].

In Fig. $\square$, we plot the results for the effective scalar-isoscalar 3NF at $\left(t-t_{0}\right) / a=8$. Here, we include $r_{2}$-independent shift by energies, $\delta_{E} \simeq 5 \mathrm{MeV}$, which is determined by long-range behavior of potentials (2NF and effective $2 \mathrm{NF}$ in the $3 \mathrm{~N}$ system) [22]. While $\delta_{E}$ suffers from $\lesssim 10 \mathrm{MeV}$ systematic error, it does not affect the following discussions much, since $\delta_{E}$ merely serves as an overall offset. In order to check the dependence on the sink time slice, we compare $3 \mathrm{NF}$ from $\left(t-t_{0}\right) / a=8$ and 9 in Fig. [1. While the results with $\left(t-t_{0}\right) / a=9$ suffer from quite large errors, they are consistent with each other within statistical fluctuations.

Fig. $\square$ shows that $3 \mathrm{NF}$ are small at the long distance region of $r_{2}$. This is in accordance with the suppression of $2 \pi \mathrm{E}-3 \mathrm{NF}$ by the heavy pion. At the short distance region, however, an indication of 


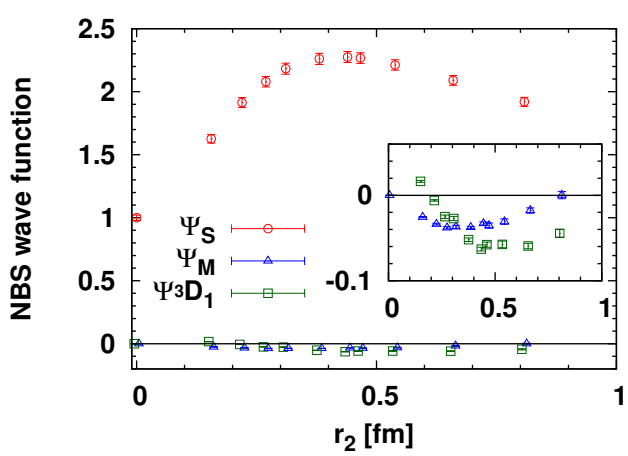

Figure 1: $3 \mathrm{~N}$ wave functions at $\left(t-t_{0}\right) / a=8$. Circle (red), triangle (blue), square (green) points denote $\psi_{S}, \psi_{M}, \psi_{{ }^{3} D_{1}}$, respectively.

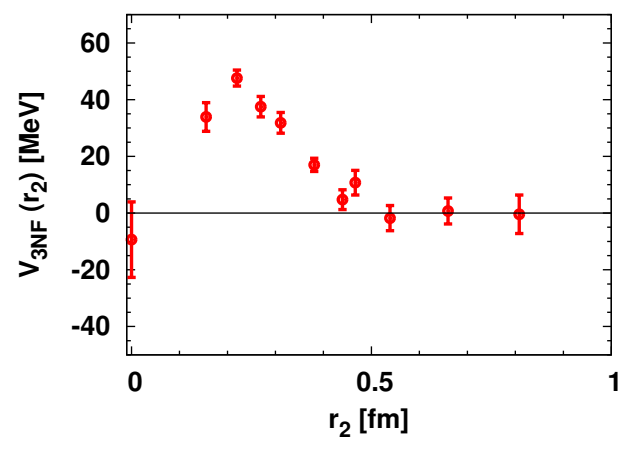

Figure 2: $\quad$ The effective scalar-isoscalar $3 \mathrm{NF}$ in the triton channel with the linear setup, obtained at $\left(t-t_{0}\right) / a=8$.

repulsive $3 \mathrm{NF}$ is observed. Note that a repulsive short-range $3 \mathrm{NF}$ is phenomenologically required to explain the properties of high density matter. Since multi-meson exchanges are strongly suppressed by the large quark mass, the origin of this short-range 3NF may be attributed to the quark and gluon dynamics directly. In fact, we recall that the short-range repulsive (or attractive) cores in the generalized two-baryon potentials are systematically calculated in lattice QCD in the flavor SU(3) limit, and the results are found to be well explained from the viewpoint of the Pauli exclusion principle in the quark level [ㄷ]]. In this context, it is intuitive to expect that the $3 \mathrm{~N}$ system is subject to extra Pauli repulsion effect, which could be an origin of the observed short-range repulsive 3NF. Further investigation along this line is certainly an interesting subject in future.

It is in order to discuss the systematic errors in lattice simulations. First, one may worry about the discretization error, since the nontrivial results are obtained at short distance. In particular, the kinetic term (Laplacian part in the Schrödinger equation) could suffer from a substantial effect, since they are calculated by the finite difference as $\nabla_{\text {std }}^{2} f(x) \equiv \frac{1}{a^{2}} \sum_{i}\left[f\left(x+a_{i}\right)+f\left(x-a_{i}\right)-2 f(x)\right]$. In order to estimate this artifact, we also analyze using the improved Laplacian operator for both of $2 \mathrm{~N}$ and $\left.3 \mathrm{~N}, \nabla_{\mathrm{imp}}^{2} f(x) \equiv \frac{1}{12 a^{2}} \sum_{i}\left[-\left(f\left(x+2 a_{i}\right)+f\left(x-2 a_{i}\right)\right)+16\left(f\left(x+a_{i}\right)+f\left(x-a_{i}\right)\right)-30 f(x)\right)\right]$. In Fig. 团, we plot the comparison between the results of $3 \mathrm{NF}$ from $\nabla_{\text {std }}^{2}$ and $\nabla_{\mathrm{imp}}^{2}$ at $\left(t-t_{0}\right) / a=8$. It is found that they are consistent with each other, and we conclude that the discretization artifact of $3 \mathrm{NF}$ in Laplacian operator is small. We, however, remark that this study probes only a part of discretization errors. Actually, the analysis with operator product expansion [28, 20] shows that 2NF of $V_{2 N}(r)$ tend to diverge as $r \rightarrow 0$, so significant discretization artifact is expected around $r=0$. Full account of the discretization artifact can be examined by an explicit lattice simulation with a finer lattice, which is currently underway.

Second, the finite volume artifact is discussed. In this simulation, three nucleons are accommodated in $(2.5 \mathrm{fm})^{3}$ spacial lattice box. We note that this is quite a large box for the heavy pion, namely, $m_{\pi} L$ is as large as 14 . Furthermore, the finite volume artifact is expected to appear mainly in large $r_{2}$ region, and we avoid it as much as possible by focusing on the short-range part of 3NF. For the relatively large $r_{2}\left(r_{2} \gtrsim 0.5 \mathrm{fm}\right)$ region, points are carefully chosen so that they are located in off-axis directions. Therefore, we expect that the finite volume artifact is not substantial in our study. Of course, a quantitative examination requires calculations with larger volumes, which we 


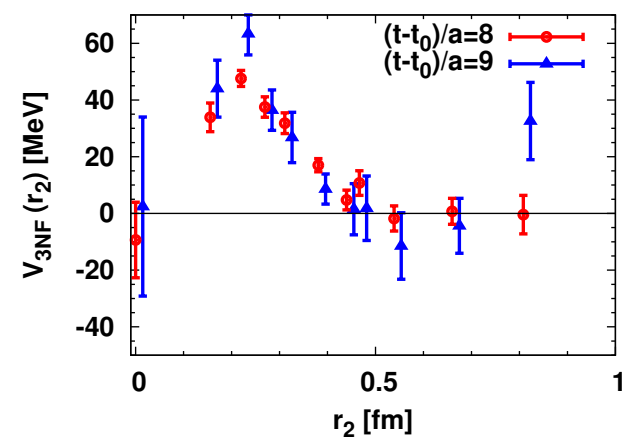

Figure 3: $3 \mathrm{NF}$ obtained at $\left(t-t_{0}\right) / a=8$ and 9 , plotted with circle (red) points and triangle (blue) points, respectively.

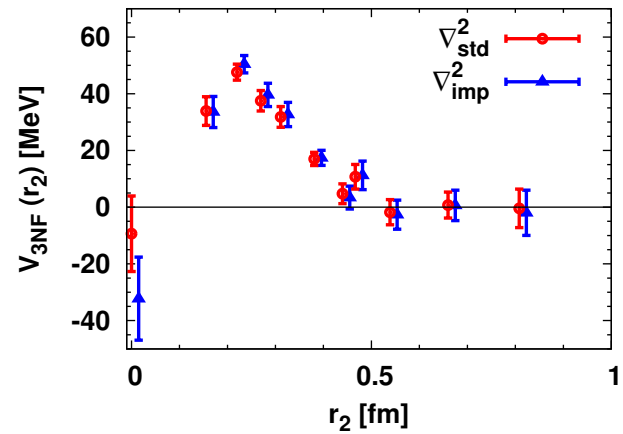

Figure 4: $3 \mathrm{NF}$ obtained at $\left(t-t_{0}\right) / a=8$. Circle (red) points are obtained from $\nabla_{\text {std }}^{2}$, and triangle (blue) points from $\nabla_{\text {imp }}^{2}$.

defer to future studies.

Third, we consider the contamination from excited states. As has been discussed, we do not observe sink time dependence for 3NF in linear setup between $\left(t-t_{0}\right) / a=8$ and 9 , nor for effective $2 \mathrm{NF}$ in the $3 \mathrm{~N}$ system as long as $\left(t-t_{0}\right) / a \geq 7$. It is, however, desirable to investigate $3 \mathrm{NF}$ in linear setup with more sink time slices. In particular, it is recently proposed [30] to utilize the time-dependent Schrödinger equation to further eliminate the excited state contamination. In order to apply this method to $3 \mathrm{NF}$, linear setup calculations at additional sink time slices are in progress.

Finally, quark mass dependence of $3 \mathrm{NF}$ is certainly an important issue, since the lattice simulations are carried out only at single large quark mass. In the case of $2 \mathrm{NF}$, short-range cores have the enhanced strength and broaden range by decreasing the quark mass [ㅍ]]. We, therefore, would expect a significant quark mass dependence exist in short-range $3 \mathrm{NF}$ as well. In addition, long-range $2 \pi \mathrm{E}-3 \mathrm{NF}$ will emerge at lighter quark masses, in particular, at the physical point. Quantitative investigation through lattice simulations with lighter quark masses are currently underway.

We thank authors and maintainers of CPS++[B]]. We also thank CP-PACS Collaboration and ILDG/JLDG [32] for providing gauge configurations. The numerical simulations have been performed on Blue Gene/L at KEK, T2K at University of Tsukuba and SR16000 at YITP in Kyoto University. This research is supported in part by MEXT Grant-in-Aid (20340047, 22540268), Scientific Research on Innovative Areas (20105001, 20105003, 21105515), Specially Promoted Research (13002001), JSPS 21.5985 and HPCI PROGRAM, the Large Scale Simulation Program of KEK (09-23, 09/10-24) and the collaborative interdisciplinary program at T2K-Tsukuba (09a11, 10a-19).

\section{References}

[1] S. C. Pieper, Riv. Nuovo Cim. 31, 709 (2008) [arXiv:0711.1500 [nucl-th]].

[2] T. Otsuka, T. Suzuki, J. D. Holt, A. Schwenk and Y. Akaishi, Phys. Rev. Lett. 105, 032501 (2010) [arXiv:0908.2607 [nucl-th]].

[3] A. Akmal, V. R. Pandharipande and D. G. Ravenhall, Phys. Rev. C58, 1804 (1998) [nucl-th/9804027]. 
[4] S. Nishizaki, T. Takatsuka and Y. Yamamoto, Prog. Theor. Phys. 108, 703 (2002).

T. Takatsuka, S. Nishizaki and R. Tamagaki, Prog. Theor. Phys. Suppl. 174, 80 (2008).

[5] T. Furumoto, Y. Sakuragi and Y. Yamamoto, Phys. Rev. C 80, 044614 (2009) [Erratum-ibid. C 82, 029908 (2010)].

[6] K. Sekiguchi et al., Phys. Rev. C 83, 061001 (2011) [arXiv:1106.0180 [nucl-ex]].

[7] J. Fujita and H. Miyazawa, Prog. Theor. Phys. 17, 360 (1957).

[8] S. C. Pieper, V. R. Pandharipande, R. B. Wiringa and J. Carlson, Phys. Rev. C 64, 014001 (2001) [arXiv:nucl-th/0102004].

[9] R. Machleidt and D. R. Entem, Phys. Rept. 503, 1 (2011) [arXiv:1105.2919 [nucl-th]] and references therein.

[10] T. Yamazaki, Y. Kuramashi and A. Ukawa, [PACS-CS Collab.], Phys. Rev. D81, 111504 (2010) [arXiv:0912.1383 [hep-lat]].

[11] S. R. Beane et al., Phys. Rev. D 80, 074501 (2009) [arXiv:0905.0466 [hep-lat]].

[12] N. Ishii, S. Aoki and T. Hatsuda, Phys. Rev. Lett. 99, 022001 (2007) [nucl-th/0611096].

[13] S. Aoki, T. Hatsuda and N. Ishii, Prog. Theor. Phys. 123, 89 (2010) [arXiv:0909.5585 [hep-lat]].

[14] H. Nemura, N. Ishii, S. Aoki and T. Hatsuda, Phys. Lett. B673, 136 (2009) [arXiv:0806.1094 [nucl-th]].

[15] T. Inoue et al. [HAL QCD Collab.], Prog. Theor. Phys. 124, 591 (2010) [arXiv:1007.3559 [hep-lat]].

[16] K. Sasaki [HAL QCD Collab.], PoS LATTICE2010, 157 (2010) [arXiv:1012.5685 [hep-lat]].

[17] T. Inoue et al. [HAL QCD Collab.], Phys. Rev. Lett. 106, 162002 (2011) [arXiv:1012.5928 [hep-lat]].

[18] S. Aoki et al. [HAL QCD Collab.], Proc. Jpn. Acad. Ser. B 87, 509 (2011) [arXiv:1106.2281 [hep-lat]].

[19] Y. Ikeda [HAL QCD Collab.], in these proceedings, arXiv:1111.2663 [hep-lat].

[20] T. Doi [HAL QCD Collab.], PoS LATTICE2010, 136 (2010) [arXiv:1011.0657 [hep-lat]].

[21] T. Doi [HAL QCD Collab.], AIP Conf. Proc. 1388, 636 (2011) [arXiv:1105.6247 [hep-lat]].

[22] T. Doi et al. [HAL QCD Collab.], arXiv:1106.2276 [hep-lat].

[23] T. Doi [HAL QCD Collab.], Proc. of the 19th Particles and Nuclei International Conference (PANIC11), arXiv:1109.4748 [hep-lat].

[24] K. Murano, N. Ishii, S. Aoki and T. Hatsuda, Prog. Theor. Phys. 125, 1225 (2011) [arXiv:1103.0619 [hep-lat]].

[25] S. Aoki, arXiv:1008.4427 [hep-lat].

[26] K. Murano [HAL QCD Collab.], in these proceedings.

[27] A. Ali Khan et al. [CP-PACS Collab.], Phys. Rev. D 65, 054505 (2002) [E: D 67, 059901 (2003)].

[28] S. Aoki, J. Balog and P. Weisz, JHEP 1005, 008 (2010) [arXiv:1002.0977 [hep-lat]].

[29] S. Aoki, J. Balog and P. Weisz, JHEP 1009, 083 (2010) [arXiv:1007.4117 [hep-lat]].

[30] N. Ishii [HAL QCD Collab.], in these proceedings.

[31] Columbia Physics System (CPS), "http://qcdoc.phys.columbia.edu/cps.html"

[32] "http://www.lqcd.org/ildg", "http://www.jldg.org" 